\title{
Article
}

\section{The outer-connected vertex edge domination number in Lexicographic product graphs}

\author{
Opeyemi Oyewumi ${ }^{1, *}$, Abolape Deborah Akwu ${ }^{2}$ and Obakpo Johnson Ben ${ }^{3}$ \\ 1 General Studies Department, Air Force Institute of Technology, Kaduna, Nigeria.; \\ fopeyemioluwaoyewumi@gmail.com \\ 2 Department of Mathematics, Federal University of Agriculture, Makurdi, Nigeria.; abolaopeyemi@yahoo.co.uk \\ 3 Department of Mathematics, Federal University Wukari, Nigeria.; benjohnsonnig@yahoo.com \\ * Correspondence: fopeyemioluwaoyewumi@gmail.com
}

Received: 03 February 2019; Accepted: 21 February 2019; Published: 10 May 2019.

\begin{abstract}
An outer-connected vertex edge dominating set (OCVEDS) for an arbitrary graph $G$ is a set $D \subset$ $V(G)$ such that $D$ is a vertex edge dominating set and the graph $G \backslash D$ is connected. The outer-connected vertex edge domination number of $G$ is the cardinality of a minimum OCVEDS of $G$, denoted by $\gamma_{v e}^{o c}(G)$. In this paper, we give the outer-connected vertex edge dominating set in lexicographic product of graphs.
\end{abstract}

Keywords: Outer-connected vertex edge domination number, Lexicographic product of graphs.

MSC: 05C05,05C69.

\section{Introduction}

$\mathbf{L}$ et $G=(V, E)$ be a simple connected graph. The length of the shortest $x-y$ path in $G$ is the distance between $x$ and $y$ denoted by $d(x, y)$ and $\max \{d(x, y): x, y \in V(G)\}$ is the diameter of $G$ denoted by $\operatorname{diam}(G)$. Let $P_{n}, C_{n}, K_{n}, W_{n}$ and $S_{n}$ denote the path of length $n$, cycle of length $n$, complete graph of length $n$, wheel of length $n$ and star graph of length $n$ respectively. A subset $D \subset V(G)$ is a dominating set (DS), of a graph $G$ if every vertex of $V(G) \backslash D$ has a neighbor in $D$. The domination number of a graph $G$, denoted by $\gamma(G)$, is the minimum cardinality of a dominating set of $G$, see [1]. A vertex $v \in V(G)$ dominates an edge $e \in E(G)$ if $v$ is incident with $e$ or with an edge adjacent to $e$. The vertex-edge dominating set of $G$ was introduced in [2] as the subset $D \subset V(G)$ if every edge of $G$ is vertex-edge dominated by a vertex in $D$. The vertex-edge domination number of a graph $G$ is the minimum cardinality of a vertex-edge dominating set of $G$ and it is denoted by $\gamma_{v e}(G)$. Further studies of vertex-edge domination in graphs can be found in [3-5].

A subset $D$ of $V(G)$ is an outer-connected dominating set of a graph $G$ if $D$ is a dominating set and $G \backslash D$ is connected. In [6], the outer-connected domination number of a graph $G$ was given as the outer-connected dominating set of $G$ with minimum cardinality and is denoted by $\gamma_{c}(G)$. Vizing [7] posed a conjecture concerning the domination number of the Cartesian product graphs. He showed that $\gamma(G) \gamma(H) \leq \gamma(G \times H)$. For a survey of domination in Cartesian products, see [8]. In [9], the notion of outer-connected vertex edge dominating set was introduced. A subset $D$ of $V(G)$ is an outer-connected vertex-edge dominating set (OCVEDS) of $G$ if $D$ is a vertex edge dominating set of $G$ and the graph $G \backslash D$ is connected. The outer connected vertex-edge domination number of a graph $G$, denoted by $\gamma_{v e}^{o c}(G)$, is the minimum cardinality of an outer connected vertex edge dominating set of $G$. The outer connected vertex edge domination number in Cartesian product of graphs has been given in [10].

The rest of this paper is organized as follows. In next Section 2, we give definition of lexicographic product and some known results. In Section 3, we give the outer-connected vertex edge domination number of the product $P_{m} \circ H$ and $C_{m} \circ H$ and the outer-connected vertex edge domination number of the product $G \circ H$ where $\gamma_{v e}^{o c}(G)=1$ and Section 4 contains concluding remarks. 


\section{Preliminaries}

Definition 1. The lexicographic product $G \circ H$ of two graphs $G$ and $H$ is a graph with vertex set $V(G) \times V(H)$ in which $\left(x_{1}, y_{1}\right)$ and $\left(x_{2}, y_{2}\right)$ are adjacent if one of the following condition holds:

1. $\left\{x_{1}, x_{2}\right\} \in E(G)$.

2. $x_{1}=x_{2}$ and $\left\{y_{1}, y_{2}\right\} \in E(H)$.

The graphs $G$ and $H$ are known as the factors of $G \circ H$.

Suppose we are dealing with $m$-copies of a graph $G$, we denote these $m$-copies of $G$ by $G^{i}$, where $i=$ $1,2,3, \ldots, m$. The Lexicographic product $G \circ H$, of graphs $G$ and $H$, can also be viewed as the graph obtained by replacing each vertex of $G$ by a copy of $H$ and every edge of $G$ by the complete bipartite graph $K_{[H],[H]}$. Henceforth, for any vertex $i \in V(G)$, we refer the copy of $H$, denoted by $H^{i}$, in $G \circ H$ corresponding to the vertex $i$ as the $i^{t h}$ copy of $H$ in $G \circ H$. The following is the outer-connected vertex edge domination number of some standard graphs that would be used in this paper.

1. [9] $\gamma_{v e}^{o c}\left(P_{n}\right)=\left\{\begin{array}{cc}1, & \text { if } n=2 \text { or } 3 \\ 2, & \text { if } n=4 \text { or } 5 \\ n-3, & \text { otherwise }\end{array}\right.$

2. [9] $\gamma_{v e}^{o c}\left(C_{n}\right)=\left\{\begin{array}{cc}1, & \text { if } n=3 \text { or } 4 \\ n-3, & \text { otherwise }\end{array}\right.$

3. [9] $\gamma_{v e}^{o c}\left(K_{n}\right)=1$, for $n \geq 1$

4. $\gamma_{v e}^{o c}\left(S_{n}\right)=1$, for $n \geq 1$

5. $\gamma_{v e}^{o c}\left(W_{n}\right)=1$, for $n \geq 1$

\section{Main results}

In this section, we give our main results.

\subsection{Outer-connected vertex edge domination number in $P_{m} \circ H$ and $C_{m} \circ H$}

Theorem 2. Let $G$ be a cycle or path of order $m$ and $H$ be a path with maximum length four, then $\gamma_{v e}^{o c}(G \circ H)=\left\lfloor\frac{m+2}{3}\right\rfloor$.

Proof. Let $D=\left\{x_{1}, x_{2}, x_{3}, \ldots, x_{j}\right\}, 1 \leq j \leq\left\lfloor\frac{m+2}{3}\right\rfloor \in \gamma_{v e}^{o c}\left(G \circ P_{n}\right)$. The graph $G \circ P_{n}$ has $m$ copies of $P_{n}$. Next we consider the following three cases.

Case 1: whenever $m \equiv 0(\bmod 3)$

Let each element of $D$ belong to any vertex of copies $P_{n}^{2}, P_{n}^{5}, P_{n}^{8}, \ldots, P_{n}^{m-1}$ respectively.

Case 2: whenever $m \equiv 1(\bmod 3)$

Let each element of $D$ belong to any vertex of copies $P_{n}^{2}, P_{n}^{5}, P_{n}^{8}, \ldots, P_{n}^{m-2}$ and $P_{n}^{m}$ respectively.

Case 3: whenever $m \equiv 2(\bmod 3)$

Again, let each element of $D$ belong to any vertex of copies $P_{n}^{2}, P_{n}^{5}, P_{n}^{8}, \ldots, P_{n}^{m}$ respectively.

Clearly, for each of the cases above, each edge in $G \circ P_{n}, 2 \leq n \leq 5$ is dominated by a vertex in $D$ and $\left(G \circ P_{n}\right) \backslash D$ is connected. Hence the proof.

Next, we have the following corollary which is immediate from Theorem 2

Corollary 3. Let $G$ be a cycle or path of order $m$, suppose we have a graph $H$ such that $\gamma_{v e}^{o c}(H)=1$, then $\gamma_{v e}^{o c}(G \circ H)=$ $\left\lfloor\frac{m+2}{3}\right\rfloor$.

Proof. The proof of this Corollary is same as that of Theorem 2.

Remark 1. Since the Wheel graph, Star graph, Complete graph and the Cycle of order 3 or 4 has their outer connected vertex edge domination number to be equal to one, then the bound mentioned in Corollary 3 covers them. 
Lemma 4. Let $G$ be a cycle or path of order three and $H=P_{n}, n \geq 6$ then $\gamma_{v e}^{o c}\left(G \circ P_{n}\right)=2$.

Proof. Consider the copies $P_{n}^{2}$ and $P_{n}^{3}$ in $G \circ P_{n}$. Assume that $D=\left\{x_{1}, x_{2}\right\} \in\left\{P_{n}^{2} \cup P_{n}^{3}\right\}$, where $x_{1} \in V\left(P_{n}^{2}\right)$ and $x_{2} \in V\left(P_{n}^{3}\right)$. It is easy to see that each edge in $G \circ P_{n}$ is dominated by a vertex in $D$. Also, $\left(G \circ P_{n}\right) \backslash D$ is connected. Therefore $\gamma_{v e}^{o c}\left(G \circ P_{n}\right)=2$, and this completes Hence the proof.

Theorem 5. Let $G$ be a cycle or path of order $m$ and $H=P_{n}, n \geq 6$, then

$\gamma_{v e}^{o c}\left(G \circ P_{n}\right)=\left\{\begin{array}{lc}\frac{m+1}{2}, & \text { if } m \text { is odd } \\ \frac{m}{2}, & \text { if } m \equiv 0(\bmod 4) \\ \frac{m+2}{2}, & \text { otherwise }\end{array}\right.$

Proof. Let $D$ be an OCVEDS of $G \circ P_{n}$. We now consider the following three cases.

Case 1: whenever $m \equiv 0$ or $3(\bmod 4)$

Applying Lemma 4 to the first three copies of $P_{n}$ we have $|D|=2$. Next, for $4 \leq q \leq m$, consider the $q^{\text {th }}$ inner copies of $P_{n}$ in the following way. Let $D^{\prime}=\left\{y_{1}, y_{2}\right\}$ such that $y_{1} \in V\left(P_{n}^{q}\right)$ if $q \equiv 2(\bmod 4)$ and $y_{2} \in V\left(P_{n}^{q}\right)$ if $q \equiv 3(\bmod 4)$.

Case 2: whenever $m \equiv 1(\bmod 4)$.

Here also, we apply Lemma 4 to the first three copies of $P_{n}$. This gives $|D|=2$. Now, for $4 \leq q \leq m-1$, consider the $q^{\text {th }}$ inner copies of $P_{n}$ as follows. Let $D^{\prime}=x \cup\left\{y_{1}, y_{2}\right\}$ such that $x \in V\left(P_{n}^{m-1}\right)$ and $y_{1} \in V\left(P_{n}^{q}\right)$ if $q \equiv 2(\bmod 4)$ and $y_{2} \in V\left(P_{n}^{q}\right)$ if $q \equiv 3(\bmod 4)$.

Case 3: whenever $m \equiv 2(\bmod 4)$

Applying Lemma 4 to the first three and last three copies of $P_{n}$ we have $|D|=4$. Next, for $4 \leq q \leq m-3$, consider the $q^{t h}$ inner copies of $P_{n}$ in the following way. Let $D^{\prime}=\left\{y_{1}, y_{2}\right\}$ such that $y_{1} \in V\left(P_{n}^{q}\right)$ if $q \equiv 2(\bmod 4)$ and $y_{2} \in V\left(P_{n}^{q}\right)$ if $q \equiv 3(\bmod 4)$.

Furthermore, notice that for each of these cases, every edge in the graph $G \circ P_{n}$ is dominated by a vertex in $\left(D \cup D^{\prime}\right)$ and $\left(D \cup D^{\prime}\right)$ is minimum. Also, $\left(G \circ P_{n}\right) \backslash\left(D \cup D^{\prime}\right)$ is connected. This completes the proof.

We now have the following corollary which is immediate from Theorem 5.

Corollary 6. Let $G$ be a cycle or path of order $m$ and $H$ be a cycle of order $n$ such that $n \geq 5$, then

$\gamma_{v e}^{o c}\left(G \circ C_{n}\right)=\left\{\begin{array}{cc}\frac{m+1}{2}, & \text { if } m \text { is odd } \\ \frac{m}{2}, & \text { if } m \equiv 0(\bmod 4) \\ \frac{m+2}{2}, & \text { otherwise }\end{array}\right.$

Proof. By applying the proof of Theorem 5 to the graph $G \circ C_{n}$, it is not hard to see that the edges of the graph $G \circ C_{n}$ is dominated by $\left(D \cup D^{\prime}\right)$ and $\left(D \cup D^{\prime}\right)$ is minimum. Also, $\left(G \circ C_{n}\right) \backslash\left(D \cup D^{\prime}\right)$ is not disconnected. Hence the proof.

\subsection{Outer-connected vertex edge domination number in $G \circ H$}

In this subsection, we provide the outer connected vertex edge domination number of the product $G \circ H$ when $\gamma_{v e}^{o c}(G)=1$.

Theorem 7. Let $H$ be a simple connected graph and $n>1$, then

$\gamma_{v e}^{o c}\left(K_{n} \circ H\right)=\left\{\begin{array}{cc}1, & \text { if } \gamma_{v e}^{o c}(H)=1 \\ 2, & \text { otherwise }\end{array}\right.$

Proof. The graph $K_{n} \circ H$ has $n$ copies of graph $H$ and the distance between any two pair of vertices of $K_{n} \circ H$ is equal to two. We now split the proof in two cases.

Case 1: whenever $\gamma_{v e}^{o c}(H)=1$ 
Let the vertex $x \in D$, where $D$ is the OCVEDS of $K_{n} \circ H$, be a vertex in any of the copy of $H$ in $K_{n} \circ H$. Clearly, $D$ dominates the graph $K_{n} \circ H$ and $\left(K_{n} \circ H\right) \backslash D$ is connected. Hence $\gamma_{v e}^{o c}\left(K_{n} \circ H\right)=1$.

Case 2: whenever $\gamma_{v e}^{o c}(H) \neq 1$

Let the vertices $x, y \in D$, where $D$ is the OCVEDS of $K_{n} \circ H$, be such that $d_{G}(x, y)=1$, i.e., $x$ and $y$ are not in the same copy of $K_{n}$ in $K_{n} \circ H$. Thus $D$ dominates the graph $K_{n} \circ H$ and $\left(K_{n} \circ H\right) \backslash D$ is connected. Hence $\gamma_{v e}^{o c}\left(K_{n} \circ H\right)=2$. The proof is complete.

Corollary 8. Suppose we have the graph $G \circ H$, such that $\gamma_{v e}^{o c}(G)=1$. If $\gamma_{v e}^{o c}(H)=\gamma_{v e}^{o c}(G)$, then $\gamma_{v e}^{o c}(G \circ H)=1$ and whenever $\gamma_{v e}^{o c}(H)>1, \gamma_{v e}^{o c}(G \circ H)=2$.

Proof. The proof of this Corollary can be drawn in the same way as that of Theorem 7.

\section{Conclusion}

We begin our conclusion with the following remark.

Remark 2. Although the lexicographic product $G \circ H$ is commutative, it is seen that the $\gamma_{v e}^{o c}(G \circ H)$ may not necessarily be equal to $\gamma_{v e}^{o c}(H \circ G)$. For instance, from Corollary 3 and Theorem $7, \gamma_{v e}^{o c}\left(C_{m} \circ K_{n}\right) \neq$ $\gamma_{v e}^{o c}\left(K_{n} \circ C_{m}\right)$, whenever $m>3$.

So far in this paper, we have provided the outer connected vertex edge domination number of the lexicographic product of two graphs. For some of the products mentioned, the outer connected vertex edge domination number of the graph $G \circ H$ depends on either the $\gamma_{v e}^{o c}(G), \gamma_{v e}^{o c}(H)$ or both.

Author Contributions: All authors contributed equally to the writing of this paper. All authors read and approved the final manuscript.

Conflicts of Interest: "The authors declare no conflict of interest."

\section{References}

[1] Haynes, T. W., Hedetniemi, S., \& Slater, P. (1998). Fundamentals of domination in graphs. CRC press.

[2] Peters, J. (1986). Theoretical and Algorithmic Results on Domination and Connectivity. Ph.D. Thesis, Clemson University.

[3] Boutrig, R., Chellali, M., Haynes, T. W., \& Hedetniemi, S. T. (2016). Vertex-edge domination in graphs. Aequationes mathematicae, 90(2), 355-366.

[4] Krishnakumari, B., Venkatakrishnan, Y. B., \& Krzywkowski, M. (2014). Bounds on the vertexÜedge domination number of a tree. Comptes Rendus Mathematique, 352(5), 363-366.

[5] Lewis, J., Hedetniemi, S. T., Haynes, T. W., \& Fricke, G. H. (2010). Vertex-edge domination. Utilitas Mathematica, 81, 193-213.

[6] Cyman, J. (2007). The outer ${ }^{1}$ connected domination number of a graph. Australasian journal of Combinatorics, 38, 35-46.

[7] Vizing, V. G. (1963). The Cartesian product of graphs. Vycisl. Sistemy, 9, 30-43.

[8] Hartnell, B., \& Rall, D. F. (1998). Domination in Cartesian products: Vizing's conjecture. Pure and Applied Mathematics-Marcel Dekker Incorporated, 209, 163-190.

[9] Krishnakumari, B., \& Venkatakrishnan, Y. B. (2018). The outer-connected vertex edge domination number of a tree. Communications of the Korean Mathematical Society, 33(1), 361-369.

[10] Akwu, A. D., Oyewumi, O., \& Ajayi, D. O. A. (2019). The outer-connected vertex edge domination number in Cartesian product graphs. Advances and Applications in Discrete Mathematics, (Accepted for publication). 